\title{
An energy aware scheme for layered chain in underwater wireless sensor networks using genetic algorithm
}

\author{
Sihem Souiki ${ }^{1}$, Sidi Mohammed Hadj Irid ${ }^{2}$, Mourad Hadjila ${ }^{3}$ \\ ${ }^{1}$ Department of Electrical Engineering, Faculty of Technology, University of Ain Temouchent, Algeria \\ ${ }^{2,3}$ Department of Telcommunications, Faculty of Technology, University of Tlemcen, Algeria
}

\section{Article Info \\ Article history: \\ Received Jul 15, 2020 \\ Revised Apr 3, 2021 \\ Accepted Apr 29, 2021}

\section{Keywords:}

Chain

Energy efficiency

Genetic algorithm

Network lifetime

Networks

Routing

Underwater wireless sensor

\begin{abstract}
Extending the network lifetime is a very challenging problem that needs to be taken into account during routing data in wireless sensor networks in general and particularly in underwater wireless sensor networks (UWSN). For this purpose, the present paper proposes a multilayer chain based on genetic algorithm routing (MCGA) for routing data from nodes to the sink. This algorithm consists to create a limited number of local chains constructed by using genetic algorithm in order to obtain the shortest path between nodes; furthermore, a leader node (LN) is elected in each chain followed by constructing a global chain containing LNs. The selection of the LN in the closest chain to the sink is as follows: Initially, the closest node to sink is elected LN in this latter because all nodes have initially the same energy value; then the future selection of the $\mathrm{LN}$ is based on the residual energy of the nodes. LNs in the other chains are selected based on the proximity to the previous LNs. Data transmission is performed in two steps: intra-chain transmission and inter-chain transmission. Furthermore, MCGA is simulated for different scenarios of mobility and density of nodes in the networks. The performance evaluation of the proposed technique shows a considerable reduction in terms of energy consumption and network lifespan.
\end{abstract}

This is an open access article under the CC BY-SA license.

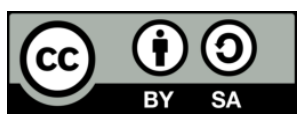

Corresponding Author:

Sihem Souiki

Department of Electrical Engineering

Faculty of Technology

University of Ain Temouchent

Algeria

Email: sihem.zineb@yahoo.com

\section{INTRODUCTION}

Water covers $72 \%$ of the surface of the globe for a total volume estimated at 1400 million $\mathrm{km}^{3}$, so it is necessary to preserve the environment, such as the collection of ocean data, pollution monitoring, disaster prevention, assisted navigation offshore exploration and tactical surveillance application. To overcome all these challenges, underwater wireless sensor network (UWSN) has become imperative [1].

In this network, diverse kinds of sensors, which can be static or dynamic, are utilized to execute cooperative actions in an area of interest. To reach this target, nodes self arrange in a very difficult environment autonomously. Furthermore, sensor nodes can communicate data collected to the base station, intermediate nodes or autonomous underwtare vehicules (AUVs). The information transmission in underwater environment may be classified on three categories: sound, electromagnetic (EM), and optical waves [2], in order to enable underwater communications through underwater sensors to exploit a variable number of applications. 
The transmission range of the radio frequency (RF) sensor presents a good performance. In other hand, underwater electromagnetic waves offer only a short distance transmission range, which makes them unusable. Many works considered using optical communication to link between the underwater sensor nodes. The main drawback of the method is that it requires both high power and high accuracy. Therefore, the best method is to use acoustic network activated by sound waves, because the acoustic signals propagate better in the underwater environement and need less energy compared to radio frequency and light signal for the same transmission range.

The sensor node in this type of networks is mainly characterized by a growing number, autonomy and movement [3]. With regard to the terrestrial wireless sensors networks, these features reveal enormous challenges such as limited bandwidth, the multi-path and fading problem which influences the underwater channel, high bit error rates, temporary losses of connectivity and long and variable propagation delay. Generally routing is the backbone for any network, because the function of routing allows the discovery and maintenance of paths. Because of the protocols incompatibility with classical sensor network [4], [5], the UWSN needs to adopt a novel energy efficient protocol, which is the aim of this paper.

Energy optimization is a serious issue in wireless sensor network deployed in aquatic environments due to the sensor nodes are supplied by batteries, which are hard to replace or recharge. However, the route construction between sensors and transmission of received data to the base station impose an energy optimized routing protocols. The design of new power efficient routing protocols for UWSN remains always a very challenging mission [6]. Especially the existing terrestrial routing protocols created are not adequate for this environment for multiple reasons: firstly water movements and other underwater activities usually cause the node mobility, secondly both radio and optical waves used in terrestrial WSN are not suitable for UWSN communication because they have great drawbacks in aquatic channel. Thus power conservation must be taken into consideration when designing any routing protocols to increase the lifetime of networks. In this context, we propose an energy efficient algorithm designed for mobile aquatic applications. The concept of our proposed algorithm consists of building several chains in multiple layers by means of genetic algorithm and elects a LN in each one. The data transmission is divided in two steps: the intra-chain transmission occurring between chain nodes that collect and transmit data to the LN followed by inter-chain transmission occurring between LNs and the sink.

The rest of this work is structured as follows: In section 2, some works related to energy efficiency routing in WSN and UWSN are briefly described. Section 3 explains in detail our approach. Section 4 is devoted to simulation results where the approaches are validated and compared to MH-FEER algorithm. Finally section 5 sets out to conclude this paper.

\section{RELATED WORKS}

Routing task in UWSN represent a primordial challenge owing to different factors that differentiate them from WSN such as sparse deployment, dynamic topology and harsh environment. Mukhtiar et al. [7] proposed an efficient and communication protocol named clustered-based energy efficient routing (CBE2R) protocol for underwater environment to control the nodes mobility and prolong the battery power of the nodes. Mukhtiar et al. proposed two surveys, the first one dedicated to routing protocols based on protocol operations for UWSN [8], and the second one dedicated to routing protocols based on node mobility for UWSN [9]. In Youngjun et al. [10], proposed a novel prototype based on heterogeneous surface gateway, which has several wireless interfaces yield to a good connectivity. This architecture can ensure a reliable connectivity between the surface gateways and control center for fairly wide range; also it can provide the sharing of low energy information between surface gateway and sensor nodes. Muhammad et al. [11] proposed the MERP protocol, which represents a bio-inspired multi-objective evolutionary routing; this protocol utilized the characteristics of the natural evolution of the multi-objective genetic algorithm in the aim to create a reliable and power efficient data collection in UWSNs. To address the problem of shortest path routing in UWSNs, Gomathi et al. [12] introduced a multiple layer routing protocol called (MRP) that can help in finding the best routing path to the destination by improving the end-to-end delay and network lifetime. Khasawneh et al. [13] proposed the location-free reliable and energy efficient pressure-based routing (RE-PBR) protocol for aquatic environment based on three parameters: depth and residual energy, link quality and reliable data delivery.

To ensure complete and reliable network coverage and achieve effective ocean monitoring of UWSNs, Liu et al. [14] proposed a distributed node deployment algorithm based on virtual forces (DABVF). In order to improve the process of node mobility, authors take the following parameters: node density, node residual energy, and node mobility. Rani et al. [15] proposed an energy efficient chain based routing protocol for UWSNs (E-CBCCP), by targeting the dynamic network. The energy of three types of nodes are considered in data transmission: cluster heads $(\mathrm{CHs})$, relay nodes (RNs) and cluster coordinators (CCOs), to 
balance the load between nodes the role of $\mathrm{CHs}$, CCOs and $\mathrm{RNs}$ changes after each time interval. A reliable multipath energy efficient routing protocol (RMEER) is designed in [16], which allows discovering the efficient path in the network, and prolonge the lifetime of nodes. The authors validated their approaches with the NS-2.30 simulator using the aquasim package. A communication techniques perspective is presented by Sandeep et al. [17] while reviewing clustering, coverage and connectivity in UWSNs.

\section{OUR PROPOSED WORK}

In this section, we briefly introduce the concept of genetic algorithm used in chains formation, and then it's presented our new proposed alogorithm.

\subsection{Genetic algorithm}

The genetic algorithms developed by Holland have interesting qualities for solving complex optimization problems [18]-[20]. Their theoretical foundations were exposed by Goldberg [21]. They attempt to simulate the evolutionary process of species in their natural environment: an artificial transposition of basic concepts of genetics and survival laws stated by Darwin. Let's recall that genetics represents an individual by a code, that is to say a set of data (called chromosomes), completely identifying the individual. Reproduction is, in this field, a random mixing of chromosomes of two individuals, giving birth to children individuals having a new genetic imprint, inherited from the parents. The genetic mutation is characterized in the genetic code of the child by the appearance of a new chromosome, non-existent in the parent individuals. This genetic phenomenon of the appearance of "mutants" is rare but allows explaining the changes in the morphology of the species, always in the direction of a better adaptation to the natural environment. The disappearance of certain species is explained by the "laws of survival" according to which only the best adapted individuals will have a sufficient longevity to generate descendants. The poorly adapted individuals will tend to disappear. It is a natural selection that leads from generation to generation to a population composed of more and more adapted individuals [22].

In our work, the aim is to form an optimal chain connecting all nodes belonging to the same level. To do this, we have inspired from the traveling salesman problem where closed chains are formed. Then we proceed to delete the longest distance between two successive nodes.

\subsection{MCGA proposed algorithm}

The deployment zone is divided into a predefined number of levels which be assumed equal to $5 \%$ of the total number of nodes. The principle of the proposed approach is broken into four phases: Chains formation phase, leader nodes election phase, data transmission phase which is itself broken in two sub phases: Intra-chain transmission and Inter-chain transmission and updating chains phase.

\subsubsection{Chains formation phase}

In each level, a shortest open chain that connects all nodes level is formed using genetic algorithm. The idea is inspired from the transport salesman problem (TSP) where a shortest closed chain is constructed then the largest distance between two successive nodes is removed in order to obtain a shortest open chain. Figure 1 illustrates the operating principles of our algorithm where the five continuous lines represent the local chains connecting nodes in each level whereas the dashed line joins the leader nodes and the sink.

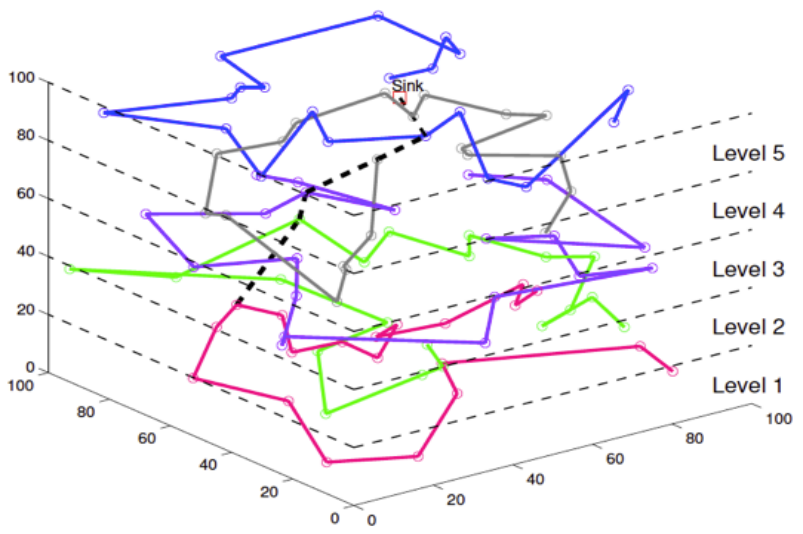

Figure 1. Principle operation of MCGA algorithm 


\subsubsection{Leader node election phase}

Once all chains are formed, we proceed to elect leader nodes. Initially, we start from the closest chain to the sink where the closest node is elected as a leader node. Leader node in the immediately next chain is chosen based on the closeness to the previous leader node and this process will be repeated for all remaining levels. This is done solely in the initially phase because at the beginning all nodes have the same amount of energy. Then leader nodes rotation is performed according to the residual energy of nodes. Thus, connecting leader nodes with the sink forms a global chain.

\subsubsection{Data transmission phase}

After chains formation and leader nodes election, sensors start data collection and transmission operation. This latter is performed in two steps: intra-chain transmission and inter-chain transmission.

a. Intra-chain transmission

In this step, there are three cases: The leader node can be the first node of chain, the last one or located between them. In the former case the leader node is the first node in the chain. As shown in Figure 2, nodes ranging from node $n-1$ to node 2 perform both transmission and reception while the first node, which is the leader node, receives data and the last node make only a transmission.

The second case is similar to the previous one but the leader node is located at the end of the chain. Therefore, the nodes ranging from node 2 to node n-1 will conduct both transmissions and receptions. The first node will perform a transmission while the latter will conduct a reception. Figure 3 depicts this situation.

The third case is illustrated in Figure 4 where the leader node is the node $\mathrm{k}$ located between the first node and the last node in the chain. Nodes ranging from node 2 to node k-1 and nodes ranging from node n- 1 to node $\mathrm{k}+1$ perform both transmission and reception whereas the first and the last nodes perform transmissions and the leader node performs two receptions.

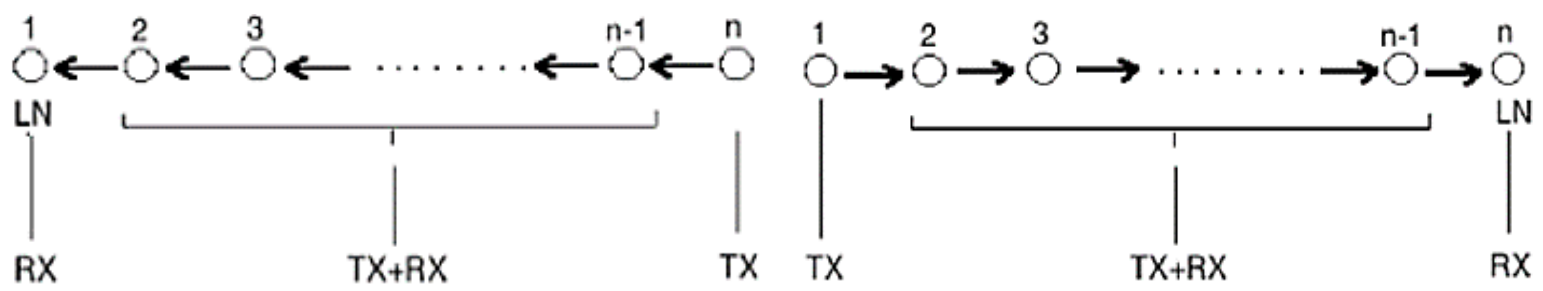

Figure 2. LN is the primary node of chain

Figure 3. LN is the end node of chain

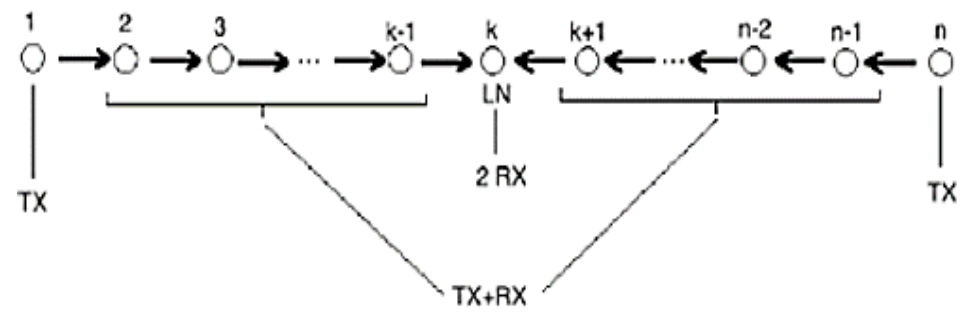

Figure 4. $\mathrm{LN}$ is inside of the chain

b. Inter-chains transmission

For gathering data at each round, leader node in the farthest level initiates by transmitting data to the next leader node, this latter receives and fuses data with its own. This process will be repeated until reaching the sink.

\subsubsection{Updating chain phase}

Figure 5(a) depicts a chain formed by eight nodes before updating, it means when all nodes have an energy level greater than 0 . If for example node 5 depletes its energy, then node 4 bypasses node 5 and sends data directly to node 6 in the next transmission see Figure 5(b). When all nodes of level depletes their energies, the number of levels decreases and the transmission is done as previously. The pseudo-code 1 summarizes the principle of MCGA algorithm. 


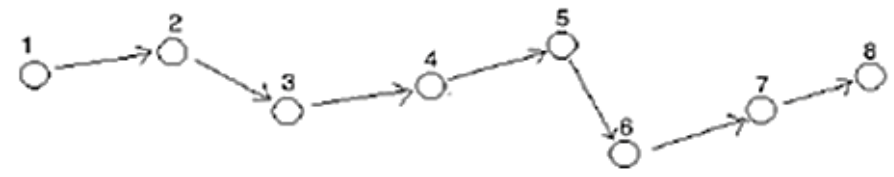

(a)

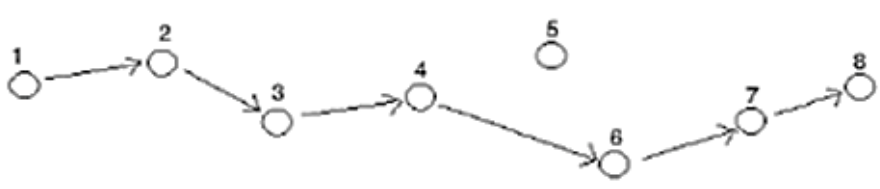

(b)

Figure 5. Updating chain; (a) a chain formed by eight nodes before updating, (b) node 5 depletes its energy

\section{PERFORMANCE EVALUATION}

In this section, it's studied the different parameters as node density and node mobility on network and their effect on the performance of our algorithm through many numerical simulations under Matlab environement. At the beginning, metrics performance and simulation method are defined. Then, the energy model is presented and finally simulation results are presented and discussed.

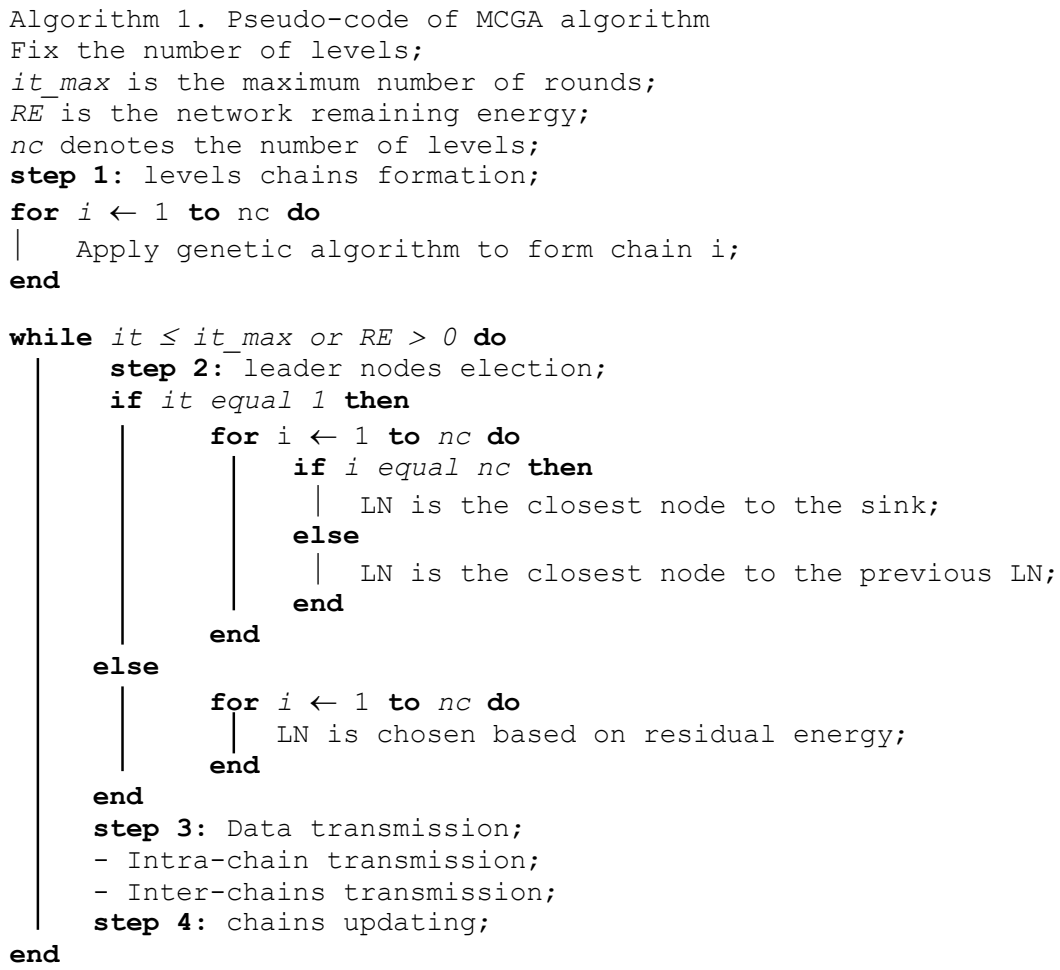

\subsection{Evaluation parameters and methodology:}

\subsubsection{Evaluation parameters}

Two metrics are necessary to perform our proposed appoach, the number of nodes alive and total energy consumption. The total energy depleted in the network is defined as the difference between the initial energy and the residual energy of all the nodes, described by the (1), (2).

$$
\begin{aligned}
& \mathrm{E}(\mathrm{i})_{\text {consumed }}=\mathrm{E}(\mathrm{i})_{\text {initial }}-\mathrm{E}(\mathrm{i})_{\text {remaining }} \\
& \mathrm{ET}_{\text {consumed }}=\sum \mathrm{E}(\mathrm{i})_{\text {consumed }}
\end{aligned}
$$

- Number of nodes alive: is the number of sensor nodes where the energy is different to 0 during (r) rounds. 


\subsection{Assumptions}

In our simulation we make the following assumptions:

- Base station and all sensor nodes are static.

- All sensor nodes are identical in terms of initial energy.

- Each sensor node knows its geographical position.

- Computing power, memory and energy are no limits for underwater base station.

- The underwater base station is located on the water surface and isn't included in sensor node deployement.

- Periodically, the received nodes receive measured environmental parameters from all sensor nodes.

- Each node has to transmit, separately, the monitoring environmental parameters to the underwater base station correctly.

The parameters setting of the simulation are shown in Table 1.

Table 1. Topology and energy parameters

\begin{tabular}{cc}
\hline Parameters & Values \\
\hline Network size & $(100 \times 100 \times 100) \mathrm{m}^{3}$ \\
Number of nodes & 100 \\
Initial energy & $10 \mathrm{~J}$ \\
Sink coordinates & $(50 \times 50 \times 50)$ \\
\hline
\end{tabular}

\subsection{Energy model}

In this work, we used the same energy model as [23], [24], which was proposed for underwater acoustic networks. According to this model, to achieve a power level $\mathrm{P}_{0}$ at a receiver at a distance $\mathrm{d}$, the transmitter power $\mathrm{E}_{\mathrm{TX}}(\mathrm{d})$ must be:

$$
\mathrm{E}_{\mathrm{TX}}(\mathrm{d})=\mathrm{P}_{0} \times \mathrm{d}^{2} \times 10^{\alpha(\mathrm{f}) / 10}
$$

where the medium absorption coefficient $\alpha(\mathrm{f})$ presented in following equation, is measured in $\mathrm{dB} / \mathrm{m}$ and is function of frequency range of interest:

$$
\alpha(\mathrm{f})=0.11 \times 10^{-3} \mathrm{f}^{2} /\left(1+\mathrm{f}^{2}\right)+44 \times 10^{-3} \mathrm{f}^{2} /\left(4100+\mathrm{f}^{2}\right)+2.75 \times 10^{-7} \mathrm{f}^{2}+3 \times 10^{-6}
$$

where $\mathrm{f}$ is the transmission carrier frequency expressed in $\mathrm{KHz}$. The reception power is assumed to $1 / 3^{\text {th }}$ of the transmission power.

\subsection{Simulation results}

The performance of the proposed algorithm is measured with the effectiveness of the energy consumption when compared with the existing MH-FEER algorithm [25]. As can be seen from Figure 6, the global energy required increases proportionally with the number of rounds. The Figure also presents that the MCGA algorithm is more economical in terms of energy and keeps the overall quantity of energy that is being used in the network. In the first, this is a lot of long distances transmission from nodes to the CHs also from CHs to the sink used in MH-FEER; these distances are avoided through the use of chain concept. Secondly, the amount of data received by the leader decreases dramatically. In the case of MH-FEER algorithm, for a cluster containing 10 nodes, the $\mathrm{CH}$ receives 10 messages, while for the same scenarion in the MCGA algorithm the leader receives only two messages.

From the simulation results shown in Figures 7 and 8, we can notice that the first node exhaust its energy in MH-FEER after 31 rounds while in MCGA algorithm the first node dies after 120 rounds. We also see that the last node dies in MH-FEER protocol after 241 rounds while in the proposed algorithm the last node dies after 296 rounds. Consequently, we can show that MCGA achieves better load balance and energy efficiency compared to MHFEER. MCGA offers a longer lifespan than MH-FEER by more than $18.58 \%$. Also, MCGA increases the lifespan and quality of the network by distributing the energy load evenly among all nodes.

\subsection{Impact of density and mobility of nodes}

Figures 9 and 10 show the density effect on the performance of our proposed algorithm. It's considered a number node variation between 100 and 300 and all nodes are mobile and have the same speed. It's shown, respectively, the effect of number of rounds under different nodes density on the total energy consumption and the nodes alive. It's seen that when the number of nodes increases, these nodes contribute in the transmission of packets and the total power consumption increase too. In other hand, the number of 
nodes alive stays stable during a number of iterations then decrease quickly. In the same way, we change the speed of the mobility of nodes from $1 \mathrm{~m} / \mathrm{s}$ to $3 \mathrm{~m} / \mathrm{s}$ and set the number of nodes to 100 . Figures 11 and 12 depict, respectively, the impact of the speed of nodes on the total energy consumption and the number of rounds under different nodes density on the nodes alive.

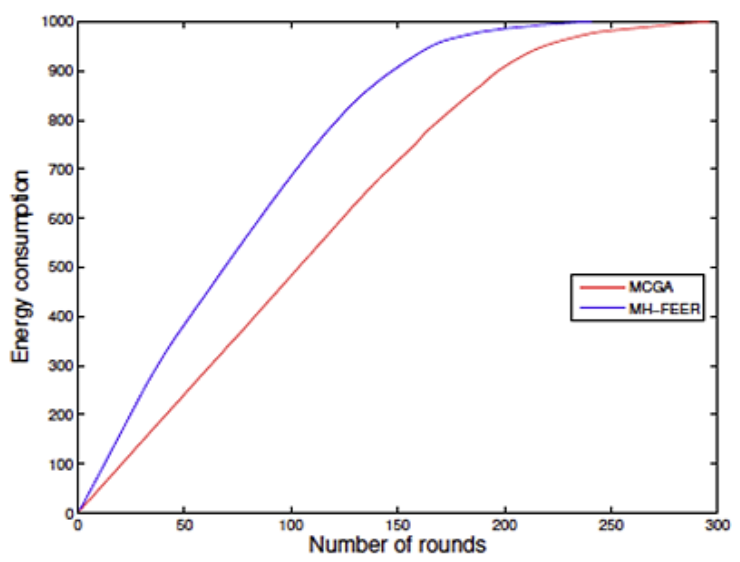

Figure 6. Total energy consumption vs. number of rounds

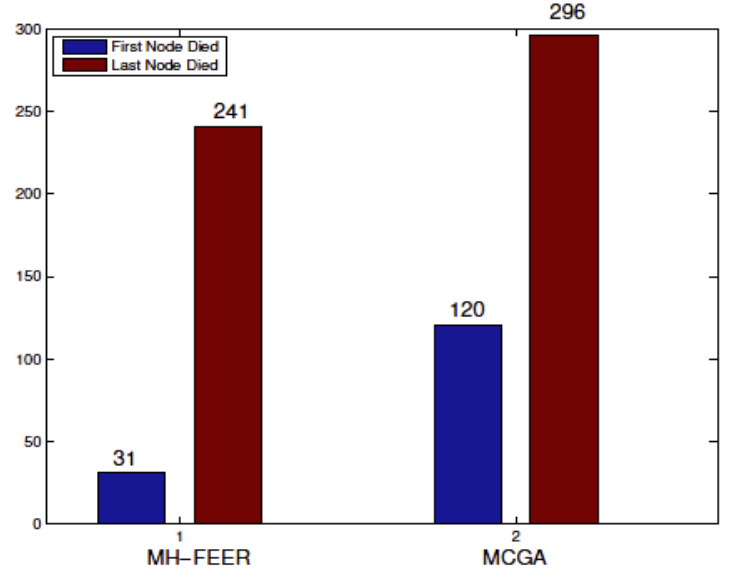

Figure 8. First and last nodes died in the network

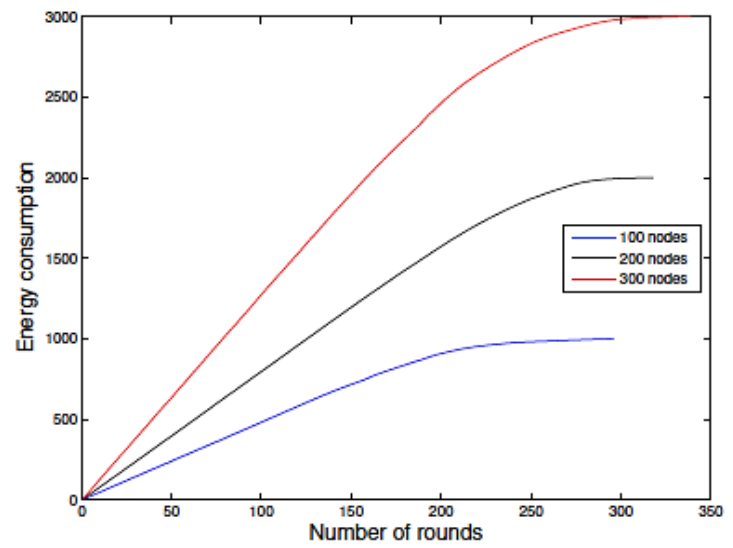

Figure 9. Total energy consumption vs. number of rounds under different nodes density

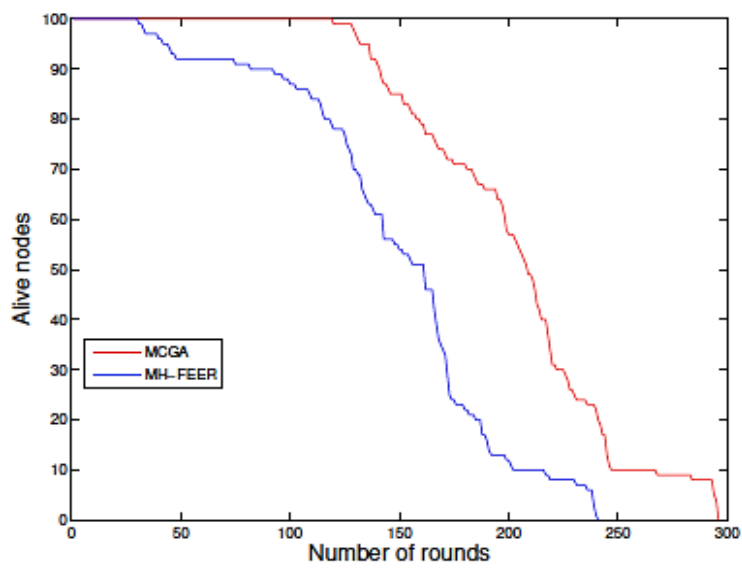

Figure 7. Nodes alive vs. number of rounds

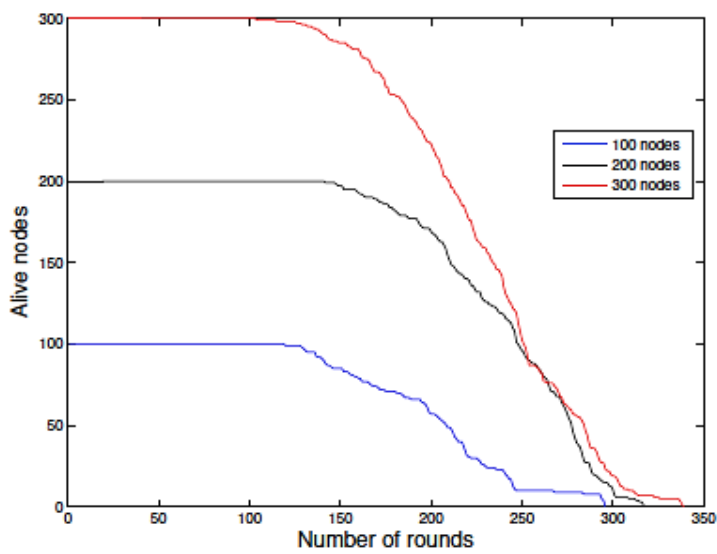

Figure 10. Nodes alive vs. number of rounds under different nodes density 


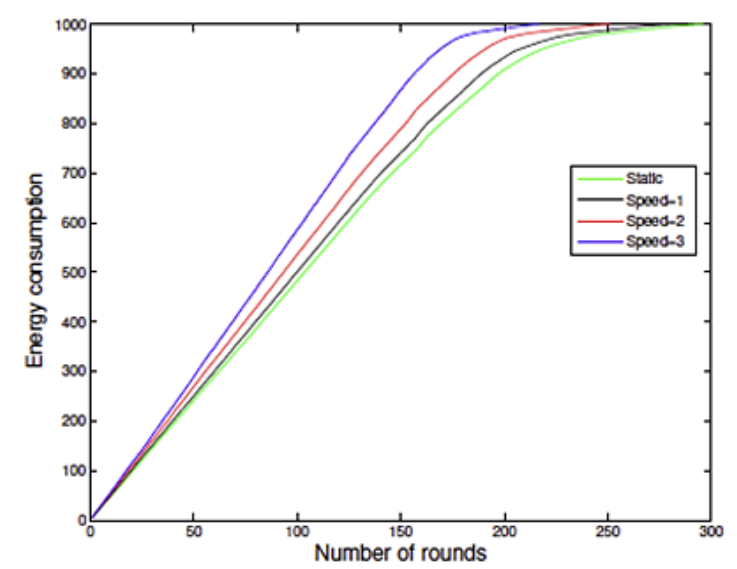

Figure 11. Total energy consumption vs. number of rounds under different mobility

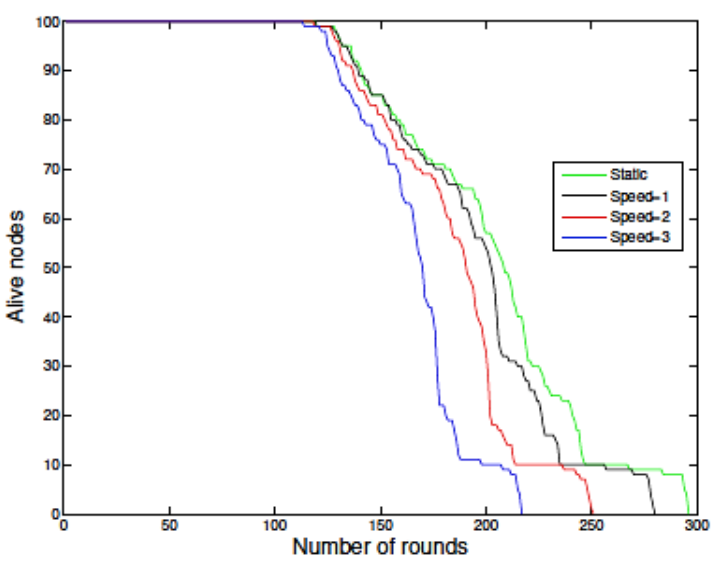

Figure 12. Nodes alive vs. number of rounds under different nodes mobility

\section{CONCLUSION AND FUTURE WORKS}

In this paper, we first discussed the issues presented by the harsh underwater environment, and the challenges associated with underwater wireless routing using acoustic communications. We then presented a chain based routing scheme that exploits the genetic algorithm to construct the chains in different levels. The simulations carried out demonstrate the scalability and effectiveness of the proposed scheme under different mobility velocities. MCGA achieves effective energy consumption in networks, consequently extending the networks longevity. As part of our ongoing work, other parameters will be introducing to elect LNs in each level such as the distance to the previous leader node. Moreover, we only evaluated our protocol with two evaluation parameters: total energy consumption and number of nodes alive, as perspectives we aim introduce the latency metric in future works.

\section{REFERENCES}

[1] L. Liu, S. Zhou, and J. H. Cui, "Prospects and Problems of Wireless Communication for Underwater Sensor Networks," Wireless Communications and Mobile Computing, vol. 8, no. 8, pp. 977-994, 2008, doi: 10.1002/wcm.654.

[2] I. F. Akyildiz, D. Pompili, and T. Melodia, "Underwater acoustic sensor networks: research challenges," Ad Hoc Networks, vol. 3, no. 3, pp. 257-279, 2005, doi: 10.1016/j.adhoc.2005.01.004.

[3] D. Makhija, P. Kumaraswamy, and R. P. Roy, "Challenges and design of mac protocol for underwater acoustic sensor networks," 2006 4th International Symposium on Modeling and Optimization in Mobile, Ad Hoc and Wireless Networks, Boston, MA, USA, 2006, pp. 1-6, doi: 10.1109/WIOPT.2006.1666499.

[4] H., Mourad, G., Hervé, F., Mohammed, "A chain-based routing protocol to maximize the lifetime of wireless sensor networks,” Wireless Sensor Network, vol. 5, no. 5, pp. 116-120, 2013, doi: 10.4236/wsn.2013.55014.

[5] M. Hadjila, H. Guyennet and M. Feham, "A Hybrid Cluster and Chain-Based Routing Protocol for Lifetime Improvement in WSN," Int. Conf. on Wired/Wireless Internet Communications, vol. 8458, pp. 257-268, 2014.

[6] R. B. Manjula, and S. M. Sunilkumar, "Issues in Underwater Acoustic Sensor Networks," International Journal of Computer and Electrical Engineering (IJCEE), vol. 3, no. 1, pp. 101-110, 2011, doi: 10.7763/IJCEE.2011.V3.299.

[7] A. Mukhtiar, S. Mazleena, and C. M. Ibrahim, "CBE2R: clustered-based energy efficient routing protocol for underwater wireless sensor network," International Journal of Electronics, vol. 105, no. 11, pp. 1916-1930, 2018, doi: 10.1080/00207217.2018.1494323.

[8] A., Mukhtiar, S., Mazleena, and Channa, M. Ibrahim, "Routing protocols based on protocol operations for underwater wireless sensor network: A survey," Egyptian Informatics Journal, vol. 19, no 1, pp. 57-62, 2018, doi: 10.1016/j.eij.2017.07.002.

[9] A., Mukhtiar, S., Mazleena, and Channa, M. Ibrahim, "Routing protocols based on node mobility for Underwater Wireless Sensor Network (UWSN): A survey," Journal of Network and Computer Applications, vol. 78, pp. 242-252, 2017, doi: 10.1016/j.jnca.2016.10.022.

[10] J. O., Youngjun and L. I. M., Sangsoon, "Design and implementation of heterogeneous surface gateway for underwater acoustic sensor network," International Journal of Electrical and Computer Engineering (IJECE), vol. 9, no 2, pp. 1226-1231, 2019, doi: 10.11591/ijece.v9i2.pp1226-1231.

[11] F. Muhammad, N. Md Asri, and V. C. Gungor, "Energy efficient multi-objective evolutionary routing scheme for reliable data gathering in Internet of underwater acoustic sensor networks," Ad Hoc Networks, vol. 93, 2019, Art. No. 101912, doi: 10.1016/j.adhoc.2019.101912.

[12] R. M. Gomathi, and J. M. L. Manickam, "Energy efficient shortest path routing protocol for underwater acoustic wireless sensor network," Wireless Personal Communications, vol. 98, no. 4, pp. 843-856, 2018, doi: 10.1007/s11277-017-4897-5. 
[13] A. Khasawneh, M. S. B. Abd Latiff, O. Kaiwartya, and H. Chizari, "A reliable energy-efficient pressure-based routing protocol for underwater wireless sensor network," Wireless Networks, vol. 24, no. 6, pp. 2061-2075, 2018, doi: 10.1007/s11276-017-1461-x.

[14] C. Liu, Z. Zhao, W. Qu, T. Qiu, and A. K. Sangaiah, "A distributed node deployment algorithm for underwater wireless sensor networks based on virtual forces," Journal of Systems Architecture, vol. 97, pp. 9-19, 2019, doi: 10.1016/j.sysarc.2019.01.010.

[15] S. Rani, S. H. Ahmed, J. Malhotra, and R. Talwar, "Energy efficient chain based routing protocol for underwater wireless sensor networks," Journal of Network and Computer Applications, vol. 92, pp. 42-50, 2017, doi: 10.1016/j.jnca.2017.01.011.

[16] M. Ahmed, M. Salleh, M. I. Channa, and M. F. Rohani, "RMEER: Reliable Multi-path Energy Efficient Routing Protocol for Underwater Wireless Sensor Network," International Journal of Electrical \& Computer Engineering (IJECE), vol. 8, no. 6, pp. 4366-4373, 2018, doi: 10.11591/ijece.v8i6.pp4366-4373.

[17] D. N. Sandeep and V. Kumar, "Review on clustering, coverage and connectivity in underwater wireless sensor networks: a communication techniques perspective," IEEE Access, vol. 5, pp. 11176-11199, 2017, doi: 10.1109/ACCESS.2017.2713640.

[18] D. L. Shanthi, and K. Prasanna, "Energy efficient intelligent routing in WSN using dominant genetic algorithm," International Journal of Electrical and Computer Engineering (IJECE), vol. 10, no. 1, pp. 500-511, 2020, doi: 10.11591/ijece.v10i1.pp500-511.

[19] P. K. Kashyap, "Genetic-fuzzy based load balanced protocol for WSNs," International Journal of Electrical and Computer Engineering (IJECE), vol. 9, no. 2, pp. 1168-1183, 2019, doi: 10.11591/ijece.v9i2.pp1168-1183.

[20] R. Khana, H. Liu, and H. Chen, "Dynamic Optimization of Secure Mobile Sensor Networks: A Genetic Algorithm," 2007 IEEE International Conference on Communications, Glasgow, UK, 2007, pp. 3413-3418, doi: 10.1109/ICC.2007.565.

[21] D. Goldberg, "Genetic Algorithms in Search, Optimization and Machine Learning," Reading, MA: Addison-Wesley, 1989.

[22] T. Murata, and H. Ishibuchi, "MOGA: Multi-Objective Genetic Algorithms," Proceedings of 1995 IEEE International Conference on Evolutionary Computation, Perth, WA, Australia, 1995, pp. 289-294, doi: 10.1109/ICEC.1995.489161.

[23] S. Souiki, M. Hadjila, and M. Feham, "Clustering combined with bio inspired routing in underwater wireless sensor networks," IPAC '15: Proceedings of the International Conference on Intelligent Information Processing, no. 62 , 2015, pp. 1-6, doi: 10.1145/2816839.2816888.

[24] W. Pu, L. Cheng, and Z. Jun, "Distributed minimum-cost clustering protocol for underwater sensor networks (UWSNs)," 2007 IEEE International Conference on Communications, Glasgow, UK, 2007, pp. 3510-3515, doi: 10.1109/ICC.2007.580.

[25] S. Souiki, M. Hadjila, and M. Feham, "Fuzzy Based Clustering and Energy Efficient Routing for Underwater Wireless Sensor Networks," International Journal of Computer Networks and Communications (IJCNC), vol. 7, no. 2, pp. 33-44, 2015, doi: 10.5121/ijcnc.2015.7203 